\title{
Eigenpath following for systems with symmetric complex-valued stiffness matrices
}

\author{
U. Miller*, S. Bograd, A. Schmidt and L. Gaul \\ Institute of Applied and Experimental Mechanics, University of Stuttgart, Pfaffenwaldring 9, 70550 Stuttgart, \\ Germany
}

\begin{abstract}
A vibration analysis of a structure with joints is performed. The simulation is conducted with finite element software capable of performing a numeric modal analysis with hysteretic damping assumption. The joints are modeled with thin layer elements, representing dissipation and stiffness of the joints. The matrices describing the system consist of the mass, as well as real and complex-valued stiffness matrices. If the eigenvalues of this system are found in one step, due to the mode crossing occurring for the closely spaced modes, it is difficult and time consuming to assign calculated modal damping factors to the corresponding undamped eigenvalues. In order to avoid this problem, an eigenvalue following method is used. The outcome of the solution is the graphical presentation of continuous eigenvalue paths, showing the change in the eigenvalues from the undamped to the fully damped case. For every undamped eigenvalue exists its equivalent eigenfrequency and damping factor that can be used for further numerical analysis.

In scope of this article a Predictor-Corrector and a Rayleigh-Quotient Iteration algorithms are applied to the problem. The algorithms are tested specifically on the type of matrices resulting from the weakly damped hysteretic formulation arising from the simulation of metallic structures with joints.
\end{abstract}

Keywords: Hysteretic damping, complex eigenvalues, path following, complex stiffness matrix

\section{Introduction}

This article is part of an ongoing research project dealing with the modeling of damping in bolted joints [1]. The main goal of the project is to be able to predict the damping in complex assembled structures subjected to micro-slip behavior in joint areas. These type of structures usually have relatively small modal damping on the order of $0.01-1 \%$ and can have high modal density. An automotive powertrain can serve as an example of this type of structure.

Viscous damping is usually used for modeling of dissipation in the finite element vibration analysis, because there exists a relatively simple mathematical model in time and frequency domain, which is well studied and is available in most of the finite element codes. However, viscous damping is proportional to the velocity, so it increases with growing frequency, which is not a common behavior for most structures. In fact, experimental investigations have shown that joint damping is nearly frequency independent [2-4]. Similar results have been shown for material damping in metals, where the main cause of dissipation is inner friction in the material [5-7].

Another method to model vibration dissipation in structures is with hysteretic damping which is completely frequency independent. A constant damping factor (loss factor) is taken for the modeling of the dissipation in materials, and joints are modeled with thin layer elements representing equivalent dissipation and stiffness of the joints [1]. This method shows a better correlation with real life structures, however it can be used only in frequency domain, since in time domain it leads to non-causal material behavior [8,9]. Some investigations to this model have been made already and show a good correlation with experimentally determined joint parameters [10,11].

\footnotetext{
${ }^{*}$ Corresponding author. E-mail: miller@iam.uni-stuttgart.de.
} 
Since it is often needed to perform simulations in time domain, for example for transient analysis, the modal damping factors are calculated in frequency domain for each mode and then are used with the undamped eigenfrequencies and modes for further simulations. So it is necessary to have a correspondence between the undamped eigenfrequencies and appropriate modal damping factors. The problem, discussed in this article, occurs for structures with closely spaced modes, where it is possible that due to the different modal damping factors the undamped eigenfrequencies do not correspond to the damped eigenfrequencies - mode order changes, making a direct damping assignment difficult. Finding corresponding damping values for the eigenfrequencies can be done by visual comparison of the damped and undamped modes, but for complicated structures with many modes this method is quite tedious and time consuming. An alternative is to use the eigenpath following method to find the damping in the system. The results of the eigenpath calculation can be represented by a table or graphically by plotting real and imaginary components of the complex eigenvalues versus the homotopy number, which is a number between zero and one, corresponding to the undamped and to the fully damped system respectively.

There exist numerous methods for pathfollowing algorithms. Two of them - Predictor-Corrector Method and Rayleigh-Quotient Iteration - are tested in scope of this article [12]. The algorithms are implemented in Matlab and their application is shown on a 64 degree of freedom mass-spring model as well as on some simple finite element models with thin layer elements. In the second case the system matrices as well as the undamped eigenvalues and eigenvectors are exported from MSC.Nastran.

The goal is to apply these methods, generally used with viscous damping matrices, to the specific problem with hysteretic damping introduced by the thin layer elements. Issues such as convergence, parallelization possibility, and accuracy are investigated. The ultimate aim of the authors is to apply the information acquired in this project, and to implement these algorithms for finite element structures with several hundred thousand degrees of freedom.

\section{Modelling of material and joint damping}

During the calculation of vibrational characteristics of a structure with the Finite Element Method the following equation of motion for an undamped system is used

$$
M \ddot{u}+K \boldsymbol{u}=\mathbf{0},
$$

where $\boldsymbol{M}$ and $\boldsymbol{K}$ are symmetric real-valued $n \times n$ mass and stiffness matrices, and $\boldsymbol{u}$ is the displacement vector. An exponential assumption $\boldsymbol{u}_{i}=\phi_{i} e^{\lambda_{i} t}$ leads to an eigenvalue problem

$$
\left(\boldsymbol{M} \lambda_{i}^{2}+\boldsymbol{K}\right) \phi_{i}=\mathbf{0}
$$

Eigenvalues and eigenmodes can be determined by performing a numerical modal analysis with a standard finite element software. Using the principle of constant hysteresis, the damping will be incorporated into the stiffness matrix by augmenting it with the complex-valued product of experimentally determined dissipation multipliers $\alpha_{i}$ and $\beta_{i}$ for the material and the joint damping, respectively, and the associated elements stiffness matrices

$$
\boldsymbol{K}^{*}=\boldsymbol{K}+\mathrm{j} \boldsymbol{D}=\boldsymbol{K}+\mathrm{j} \sum \alpha_{i} \boldsymbol{K}_{i}^{\text {(material) }}+\mathrm{j} \sum \beta_{i} \boldsymbol{K}_{i}^{(\text {joint })} .
$$

For the considered systems, damping is low and $\alpha_{i}, \beta_{i} \ll 1$ holds. The solution of the complex-valued system

$$
\left(\boldsymbol{M} \lambda_{i}^{2}+\mathrm{j} \boldsymbol{D}+\boldsymbol{K}\right) \boldsymbol{\phi}_{i}=\left(\boldsymbol{M} \lambda_{i}^{2}+\boldsymbol{K}^{*}\right) \boldsymbol{\phi}_{i}=\mathbf{0}
$$

leads to complex eigenvalues $\lambda_{i}=\delta_{i}+\mathrm{j} \omega_{i}$ and eigenvectors $\phi_{i}=\boldsymbol{r}_{i} e^{\mathrm{j} \kappa_{i}}$. The solution vector represented by an exponential assumption may be written as

$$
\boldsymbol{u}_{i}=\phi_{i} e^{\lambda_{i} t}=\boldsymbol{r}_{i} e^{\mathrm{j} \kappa_{i}} e^{\lambda_{i} t}=\boldsymbol{r}_{i} e^{\delta_{i} t+\mathrm{j}\left(\omega_{i} t+\kappa_{i}\right)},
$$

where $\boldsymbol{r}_{i}$ is the amplitude of vibration, $\delta_{i}$ is the modal damping, $\omega_{i}$ and $\kappa_{i}$ are the frequency and the phase shift, respectively.

This modified Eq. (4) can be solved for complex eigenvalues and eigenmodes with some commercial finite elements packages, which in this case was performed with Nastran. The modal damping factors of the structure are read out from the solution. 
The thin layer elements used for the simulation of joints in the Finite Element model contain experimentally determined contact stiffness and dissipation parameters as their material properties. They are normal hexa- or pentahedral elements in which the length or width to thickness ratio can be up to 1000:1 without causing numerical problems during the calculation [13]. They are implemented to describe the local (joint) dissipation in structures and it has been shown that the simulated damping correlates better with the experimental results than traditional damping modeling methods [1].

\section{Path following}

A transient or an operational vibration analysis of mechanical systems often requires a correspondence between the damped and the undamped eigenvalues, where the modal damping factors are assigned to the undamped eigenmodes. For systems with high mode density, it is difficult to find this correspondence if the damped system is solved in one step without any relation to the undamped one.

To find this mapping between the eigenvalues, a so called homotopy parameter $\mu$ is introduced so that the solution follows the eigenvalues from the undamped system at $\mu=0$ to the damped system at $\mu=1$ [12]. Then the calculated paths can be constructed, showing clearly where the modes cross each other. In addition, the sensitivity of the calculated modal damping factors may be analyzed [14].

The system equation of motion (4) with homotopy parameter $\mu$ has the following form

$$
\left(\boldsymbol{M} \lambda_{i}^{2}+\mu \mathrm{j} \boldsymbol{D}+\boldsymbol{K}\right) \boldsymbol{\phi}_{i}=\mathbf{0}, 0 \leqslant \mu \leqslant 1,
$$

where $\lambda_{i}$ und $\phi_{i}$ are the complex eigenvalues and corresponding eigenvectors of the system which coincide with the eigenvalues and eigenvectors of the undamped system for $\mu=0[15,16]$. Now the path following, also known as the numeric continuation method is implemented. Based on the undamped system at $\mu=0$, the homotopy parameter is increased step by step and the eigenvalue problem (6) is solved. In this work, the steplength has been fixed.

There are two different methods described in this article. The first method shown - the Predictor-Corrector Method - is based on the initial prediction of the desired solution of the eigenvalue problem and then it uses Newton's method for correction to achieve appropriate accuracy. Afterward, the Rayleigh-Quotient Iteration is presented which is a widely used iterative method to compute eigenvalues and eigenvectors of symmetric systems.

The Rayleigh-Quotient Iteration as well as Newton's Method can be regarded as an extension of the Inverse Iteration, which is one of the oldest algorithms for finding eigenvalues [17-19]. Chatelin [20] and Amiraslani and Lancaster [21] compare the methods directly and show that the Rayleigh-Quotient Iteration is an extension of Newton's Method.

The convergence rate of the Inverse Iteration is linear, Newton's Method converges quadratically and the RayleighQuotient-Iteration cubically [20-22]. The three methods require the solution of a linear system of equations in each iteration which can be parallelized [22].

\subsection{Predictor-corrector method}

Because the eigenvectors can be scaled arbitrarily, the eigenvalue problem (6) is expanded by a suitable scaling equation

$$
\left[\begin{array}{c}
\left(\boldsymbol{M} \lambda_{i}^{2}+\mu \mathrm{j} \boldsymbol{D}+\boldsymbol{K}\right) \boldsymbol{\phi}_{i} \\
\boldsymbol{\phi}_{i 0}^{H} \boldsymbol{\phi}_{i}
\end{array}\right]=\left[\begin{array}{l}
\mathbf{0} \\
1
\end{array}\right]
$$

with the starting eigenvector $\phi_{i 0}$ (for $\mu=0$ ). If the eigenpair $\left(\phi_{i}, \lambda_{i}\right)$ is denoted by a vector

$$
\boldsymbol{z}_{i}=\left[\begin{array}{c}
\phi_{i} \\
\lambda_{i}
\end{array}\right]
$$

then Eq. (7) can be written as

$$
\boldsymbol{g}\left(\boldsymbol{z}_{i}(\mu), \mu\right)=\left[\begin{array}{c}
\boldsymbol{g}_{1} \\
g_{2}
\end{array}\right]=\left[\begin{array}{c}
\left(\boldsymbol{M} \lambda_{i}^{2}+\mu \mathrm{j} \boldsymbol{D}+\boldsymbol{K}\right) \boldsymbol{\phi}_{i} \\
\boldsymbol{\phi}_{i 0}^{H} \boldsymbol{\phi}_{i}-1
\end{array}\right]=\mathbf{0}
$$


This equation depends nonlinearly on $\lambda_{i}$, and is solved with the help of the Predictor-Corrector Formulation with the assumption

$$
\boldsymbol{g}\left(\boldsymbol{z}_{i}(\mu), \mu\right)=\boldsymbol{g}\left(\overline{\boldsymbol{z}}_{i}(\mu), \bar{\mu}\right)+\left.\boldsymbol{g}_{, \boldsymbol{z}}\right|_{\overline{\boldsymbol{z}}_{i, \bar{\mu}}} \Delta \boldsymbol{z}=\mathbf{0},
$$

where $\overline{\boldsymbol{z}}_{i}, \bar{\mu}$ are known values and $\circ_{, x}$ are derivatives with respect to different values of $x$ [14]. The required derivatives are

$$
\begin{aligned}
\boldsymbol{g}_{1, \boldsymbol{\phi}_{i}} & =\lambda_{i}^{2} \boldsymbol{M}+\mu \mathrm{j} \boldsymbol{D}+\boldsymbol{K} \\
\boldsymbol{g}_{1, \lambda_{i}} & =2 \lambda_{i} \boldsymbol{M} \boldsymbol{\phi}_{i} \\
g_{2, \boldsymbol{\phi}_{i}} & =\boldsymbol{\phi}_{i 0}^{\mathrm{H}} \\
g_{2, \lambda_{i}} & =0 .
\end{aligned}
$$

Differentiating Eq. (9) with respect to $\mu$ leads to

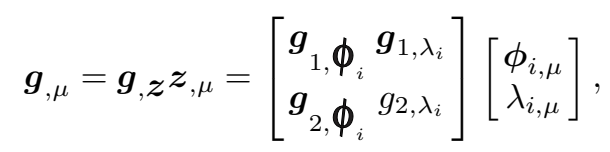

and Eq. (10) reformulated yields

$$
\left.\boldsymbol{g}_{, \boldsymbol{z}}\right|_{\overline{\boldsymbol{z}}_{i, \bar{\mu}}} \Delta \boldsymbol{z}=\left[\begin{array}{ll}
\boldsymbol{g}_{1, \boldsymbol{\phi}_{i}} & \boldsymbol{g}_{1, \lambda_{i}} \\
\boldsymbol{g}_{2, \boldsymbol{\phi}_{i}} & g_{2, \lambda_{i}}
\end{array}\right] \Delta \boldsymbol{z}=-\left[\begin{array}{l}
\boldsymbol{g}_{1} \\
g_{2}
\end{array}\right] .
$$

For the predictor step, the derivation

$$
\boldsymbol{g}_{, \mu}=\left[\begin{array}{c}
\left(2 \lambda_{i} \lambda_{i, \mu} \boldsymbol{M}+\mathrm{j} \boldsymbol{D}\right) \boldsymbol{\phi}_{i}+\left(\lambda_{i}^{2} \boldsymbol{M}+\mu \mathrm{j} \boldsymbol{D}+\boldsymbol{K}\right) \boldsymbol{\phi}_{i, \mu} \\
\boldsymbol{\phi}_{i 0}^{\mathrm{H}} \boldsymbol{\phi}_{i, \mu}
\end{array}\right]=\mathbf{0}
$$

is used, which leads to

$$
\left[\begin{array}{cc}
\boldsymbol{g}_{1, \boldsymbol{\phi}_{i}} & \boldsymbol{g}_{1, \lambda_{i}} \\
\boldsymbol{g}_{2, \boldsymbol{\phi}_{i}} & 0
\end{array}\right] \boldsymbol{z}_{, \mu}=\left[\begin{array}{c}
-(\boldsymbol{K}+\mathrm{j} \boldsymbol{D}) \boldsymbol{\phi}_{i} \\
0
\end{array}\right] .
$$

Equation (7) can be solved iteratively for the actual homotopy parameter

$$
\mu_{n+1}=\mu_{n}+h
$$

with predictor

$$
{ }^{n+1} \boldsymbol{z}_{i}={ }^{n} \boldsymbol{z}_{i}+h \cdot{ }^{n} \boldsymbol{z}_{i, \mu}
$$

calculated from Eq. (18). Then the result is corrected with Newton's Method from Eq. (16)

$$
{ }^{n+1} \boldsymbol{z}_{i}^{(t+1)}={ }^{n+1} \boldsymbol{z}_{i}^{(t)}+\Delta \boldsymbol{z}, \quad t=1,2, \ldots
$$

until the residual reaches a predefined convergence parameter.

\subsection{Rayleigh-quotient iteration}

The Rayleigh-Quotient of a vector $\phi$ is defined as [23-25]

$$
r(\phi) \equiv \frac{\phi^{T} \boldsymbol{K}^{*} \boldsymbol{\phi}}{\boldsymbol{\phi}^{T} \boldsymbol{M} \boldsymbol{\phi}}
$$




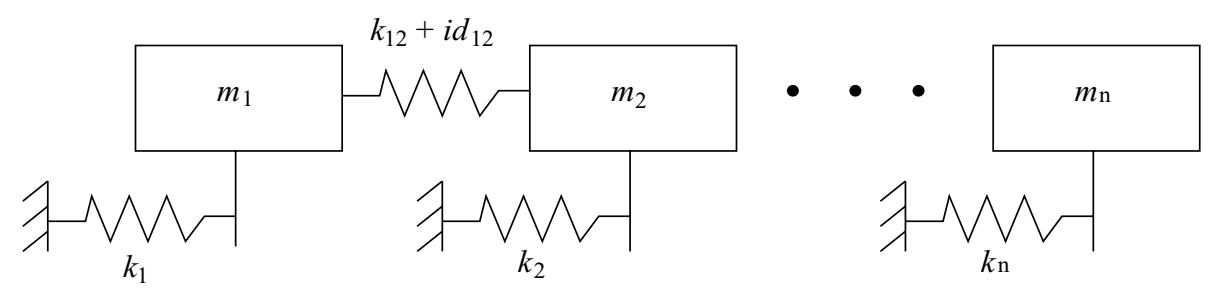

Fig. 1. Test structure: Mass spring system.

with symmetric $n \times n$ matrices $\boldsymbol{K}^{*}$ and $\boldsymbol{M}$. If $\phi_{0}$ is an approximation to an eigenvector of the generalized eigenvalue problem $\boldsymbol{K}^{*} \phi=\lambda \boldsymbol{M} \phi$, then $r\left(\phi_{0}\right)$ can be used to find an approximation to the corresponding eigenvalue $\lambda_{0}$. On the other hand, the solution of

$$
\left(\boldsymbol{K}^{*}-\lambda_{0} \boldsymbol{M}\right) \phi_{0}=\boldsymbol{b}
$$

is almost always a good choice for the eigenvector of the generalized eigenvalue problem, if there exists an approximate value $\lambda_{0}$ [23]. Also, Eq. (23) can be solved with a suitable assigned approximation to the eigenvector for the right hand side $\boldsymbol{b}$ (e.g.: undamped eigenvector for the damped problem). In case there is no approximate eigenvector available, one can choose a random vector, but this can lead to divergence and long solution times.

These two ideas are combined and adjusted to fit the available problem with symmetric real matrix $M$ and complex matrix $\boldsymbol{K}^{*}$ to the Rayleigh-Quotient Iteration algorithm. The starting vector $\phi_{0}$ from the undamped eigenvalue solution with $\left\|\phi_{0}\right\|=1$ is taken. Now iterating for $k=0,1, \ldots$ using Eq. (22) the eigenvalue

$$
\lambda_{k}=r\left(\phi_{k}\right)
$$

is found. In the next step equation

$$
\left(\boldsymbol{K}^{*}-\lambda_{k} \boldsymbol{M}\right) \boldsymbol{z}_{k+1}=\phi_{k}
$$

is solved for $\boldsymbol{z}_{k+1}$. The approximation of the eigenvector from the previous iteration, starting with $\phi_{0}$, is taken for the right hand side of the Eq. (25). Then the solution is normalized

$$
\phi_{k+1}=z_{k+1} /\left\|z_{k+1}\right\| \text {. }
$$

The norm of the computed vector $\left\|\boldsymbol{z}_{k+1}\right\|$ increases while iterating and serves as a convergence criterion.

Sometimes the algorithm has problems converging to the eigenvalue next to the starting value $\lambda_{0}$ [17]. In order to avoid this problem for the path following algorithm the eigenvector from the last homotopy step is taken as a starting vector and the number of the homotopy steps can be increased.

\section{Application and results}

\subsection{Test structures}

The algorithms are tested on several models with symmetric mass and stiffness matrices, where the size of the models varies between 64 and 38469 degrees of freedom. The smallest one consists of masses and springs with system matrices built in Matlab while the other systems are modeled using the finite element program Nastran.

The first small model is rather simple and was used for algorithm testing during the programming. It has 64 degrees of freedom consisting of a chain of masses connected by springs. In addition, each mass is connected to ground by another spring (Fig. 1). Some of the springs in the middle of the chain have a stiffness with an imaginary part $d_{i j}$ to incorporate hysteretic damping into the system.

The following parameters are chosen for the chain of masses and springs: 


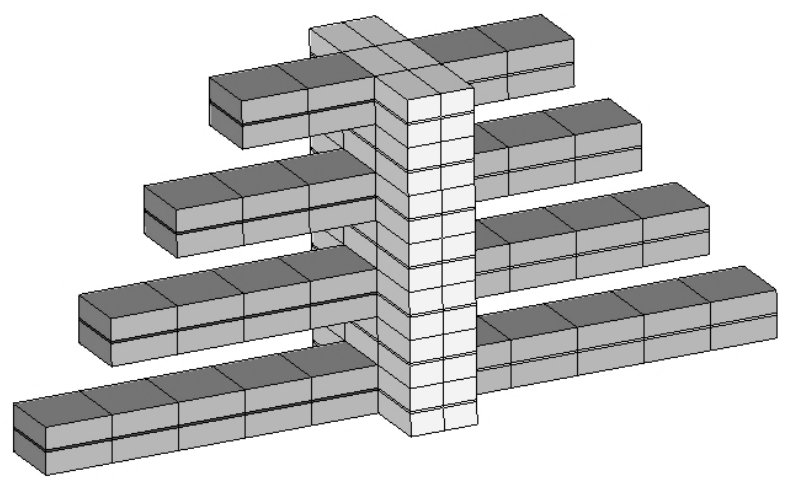

Fig. 2. Finite Element model of the tree structure (1464 degrees of freedom).
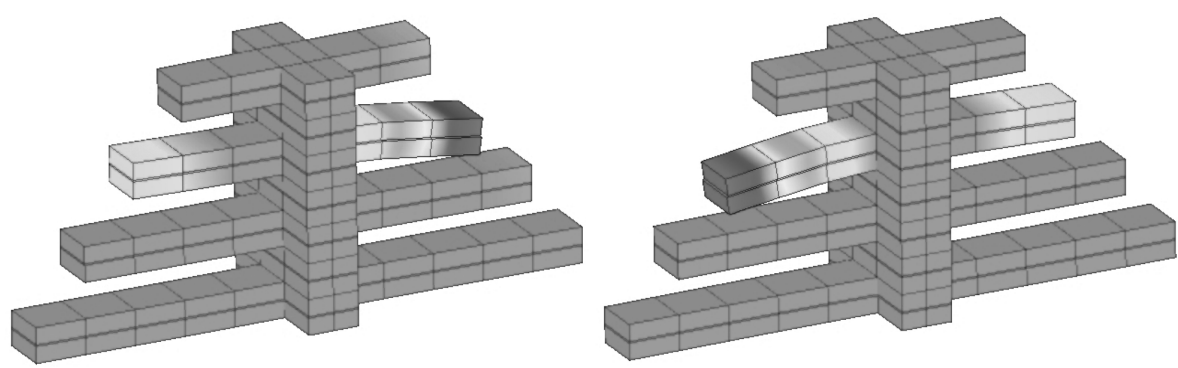

Fig. 3. Mode shape 7 (left) and 8 (right) of the tree structure.

$$
\begin{aligned}
m_{i} & =1 \mathrm{~kg}, \\
k_{i} & =1000 \mathrm{~N} / \mathrm{m}, \\
k_{i j} & =1000 \mathrm{~N} / \mathrm{m},
\end{aligned} \quad d_{i j}=\left\{\begin{array}{l}
0 N / m \text { for } i<30, j<31, \\
30 N / m \text { for } 30 \leqslant i \leqslant 35,31 \leqslant j \leqslant 36, \\
0 N / m \text { for } i>35, j>36 .
\end{array}\right.
$$

The second structure is called the tree model and shown in Fig. 2. It is modeled with steel parameters using brick elements and consists of a stem with eight attached arms. Every arm is assembled from two identical beams connected by thin layer elements to simulate joints with damping in the system. The stiffness of the thin layer elements on the left is slightly higher than on the right side whereas the damping is higher on the right side so that there are some eigenfrequencies lying close together and crossing when the damping is increased. Figure 3 shows the shapes of modes 7 and 8 with eigenfrequencies of the undamped system of $111.3 \mathrm{~Hz}$ and $115.0 \mathrm{~Hz}$, respectively.

Finally, three structures, made out of two $3 \mathrm{~mm}$ thick steel plates joined by bolts, were used for timing purposes. The joints are modeled with thin layer elements with constant damping. Since steel has very low damping and no macro-slip occurs in the joints, damping of the assembly for the first 10 modes is lower than $0.2 \%$. The structures are modeled with brick elements and have 3468, 6936 and 38469 translational degrees of freedom.

The undamped eigenvalues and eigenmodes of the four finite element models are found with Nastran and mass, stiffness, and damping matrices are exported into ASCII files which in turn are imported into Matlab in sparse format.

\subsection{Results}

An example of an eigenpath following for the tree model with 1464 degrees of freedom is shown in Fig. 4 where the eigenvalues for modes 7 until 18 are plotted. There are no mode crossings for modes 1 through 6 , hence they are omitted to make the figure more readable. The eigenvalues of modes 7 and 8 as well as of modes 16 and 17 cross each other because of the significantly different damping values, which are plotted on the right side of the figure.

The Predictor-Corrector Method needs at least 10 homotopy steps to follow the paths of the tree model correctly, i.e. every eigenvector of the damped system converges to the corresponding eigenvector of the undamped one. For the mass-spring system, the Rayleigh-Quotient Iteration needs 4 homotopy steps to find the correct eigenpaths. 

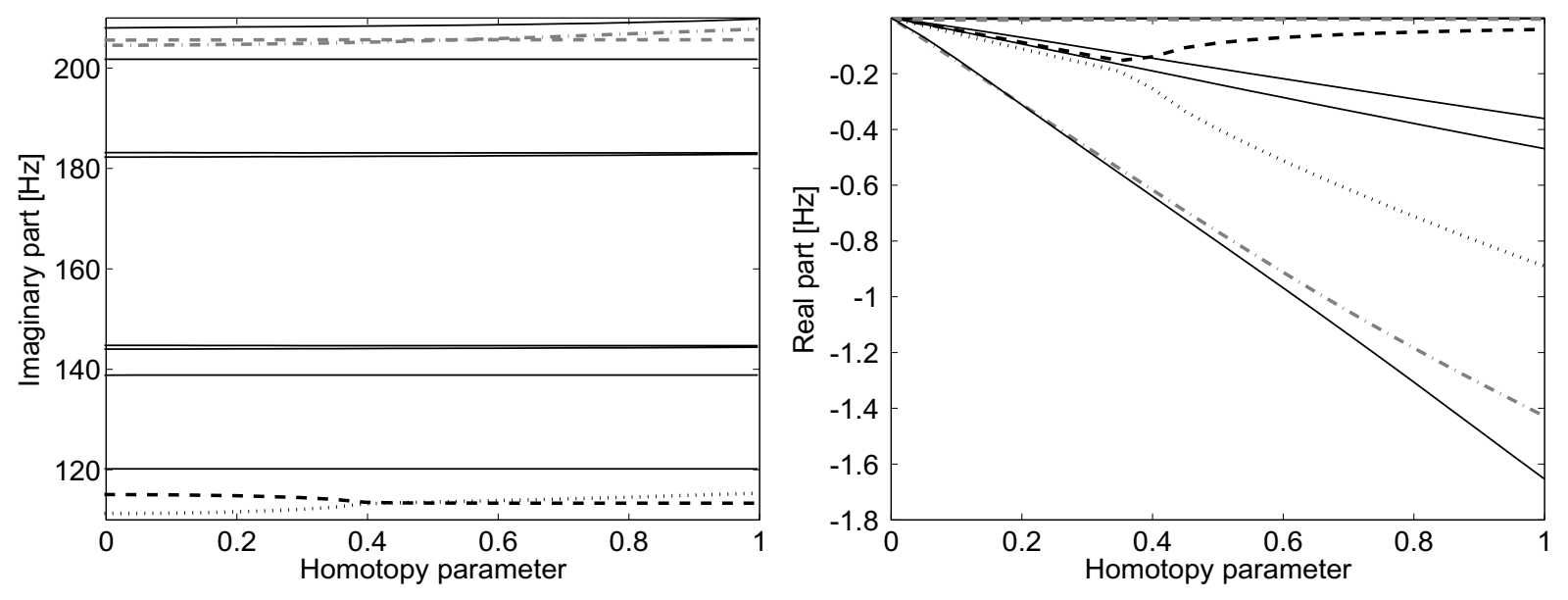

Fig. 4. Imaginary and real part of 12 eigenvalues of the tree model.

To follow the eigenpaths of the tree system, the Rayleigh-Quotient Iteration actually needs only two homotopy steps. The eigenvalue of mode 16 crosses that of mode 17 and if only one step is used, the eigenvector of mode 18 converges to the damped eigenvector of mode 16. For the other finite element models with bolted steel plates, only one step of Rayleigh-Quotient Iteration was sufficient to follow the first 20 eigenpaths.

By loosening the convergence criterion, which was set to 0.001 in all cases, both algorithms have problems converging to the right modes so that more homotopy steps are needed.

\subsection{Computational time and accuracy}

Calculations were performed on a computer with four 3.0 GHz dual core processors with 16 GB RAM. Matlab (version 7.6) automatically parallelizes the solution of linear systems so that for both algorithms all the processors were used.

For each model, 20 natural modes were calculated and the average values of the computational times related to the calculation of each natural mode are plotted in Fig. 5. For both algorithms, the lowest number of homotopy steps needed to correctly follow the eigenvalues was used, i.e. 10 steps for the Predictor-Corrector Method and 4 steps for the Rayleigh-Quotient Iteration. As a reference, the Rayleigh-Quotient Iteration was also performed with 10 steps.

The Rayleigh-Quotient Iteration is faster, since it needs fewer homotopy steps. If it has to calculate 10 homotopy steps, it is still a little bit faster than the Predictor-Corrector Method, because it has the better convergence rate and needs less iteration steps in order to converge. No relevant difference in time is noticed for the calculation of the eigenvalues for closely spaced modes and for those located far apart from each other.

Apart from the differences resulting from non-identical matrix operations, an important role in computational time plays the accuracy of the results, which in turn is dependent on the convergence criterion - residual error and number of homotopy steps needed for the problem to converge and to give appropriate accuracy.

The benchmark solution for the finite element models is performed with the complex eigenvalue solver in Nastran. Both algorithms showed nearly identical results with marginal difference between the Nastran and Matlab values. Experimental investigations for the largest model were made as well and they correlate well with the simulation results [1].

\section{Conclusions}

This article gives a short overview and comparison of two eigenvalue following algorithms: Predictor-Corrector and Rayleigh-Quotient Iteration. Both algorithms are applied to a weakly damped finite element model with hysteretic damping. They achieve comparable results in terms of accuracy, however Rayleigh-Quotient Iteration converges faster and requires lower number of homotopy steps. 


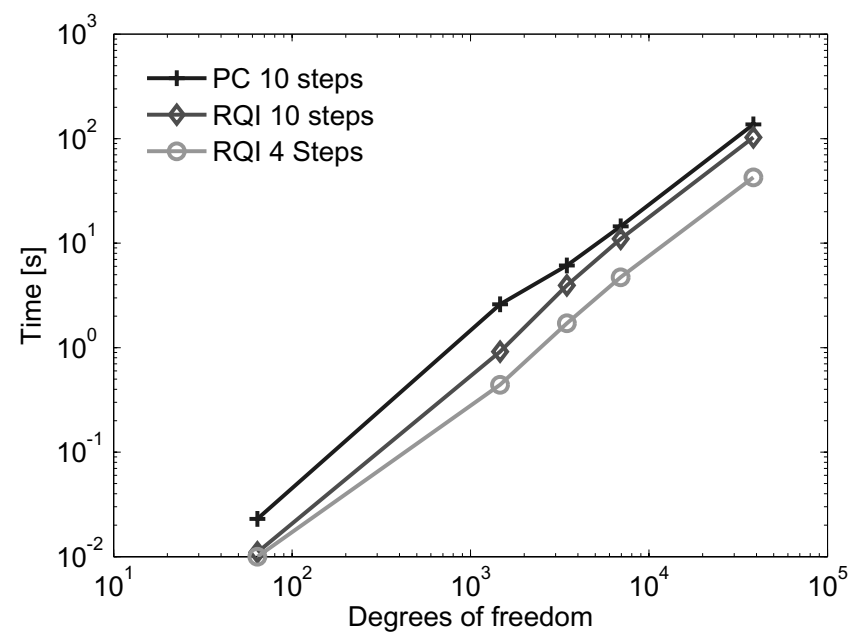

Fig. 5. Computional times for one mode for the models with different degrees of freedom.

The next steps in the project will concentrate on modification of algorithms. In particular - implementation of adaptive homotopy step-length and introduction of block variant algorithms to ensure the correct path following when mode crossings occur. In the end the algorithms will be implemented in a higher level programming language like Fortran or $\mathrm{C}++$.

\section{Acknowledgments}

The support of the project "Experimentelle Ermittlung von Kennwerten zur Werkstoff- und F ügestellendämpfung sowie deren Berücksichtigung in Finite-Elemente-Berechnungen" by the Forschungsvereinigung Verbrennungskraftmaschinen (FVV) and Deutsche Forschungsgemeinschaft (DFG) which enabled us to perform these investigations is gratefully acknowledged. We would also like to thank Dr.-Ing. Nils Wagner for his help with implementation of the Predictor-Corrector Method.

\section{References}

[1] L. Gaul, A. Schmidt and S. Bograd, Experimentelle Ermittlung von Kennwerten zur Werkstoff- und Fugestellendämpfung sowie deren Berücksichtigung in Finite-Elemente-Berechnungen. Forschungsvereinigung Verbrennungskraftmaschinen (FVV) Heft 859, Frankfurt, 2008.

[2] D. Ewins, Modal Testing: Theory, Practice and Application, (2nd edition), Research Studies Press, 2000

[3] L. Garibaldi and H.N. Onah, Viscoelastic Material Damping Technology, Beccis Osiride, 1996.

[4] L. Gaul and R. Nitsche, The role of friction in mechanical joints, Applied Mechanics Reviews 54 (2001), 93-106.

[5] R.S. Lakes. Viscoelastic Solids. CRC Press, 1999.

[6] A. Schmidt and L. Gaul, On the numerical evaluation of fractional derivatives in multi-degree-offreedom systems, Signal Processing 86 (2006), 2592-2601.

[7] A. Wineman and K.R. Rajagopal, Mechanical Response of Polymers, University Press, 2000.

[8] S. Crandall, The role of damping in vibration theory, Journal of Sound and Vibration 11 (1970), 3-18.

[9] J.A. Inaudi and N. Makris, Time-domain analysis of linear hysteretic damping, Earthquake Engineering and Structural Dynamics 25 (1996), 529-545.

[10] D.J. Henwood, Approximating the hysteretic damping matrix by a viscous matrix for modeling in the time domain, Journal of Sound and Vibration 254 (2002), 575-593.

[11] A.D. Nashif, D.I.G. Jones and J.P. Henderson, Vibration Damping, John Wiley and Sons, 1985.

[12] E.L. Allgower and K. Georg, Introduction to Numerical Continuation Methods, SIAM, 2003.

[13] C.S. Desai, M.M. Zaman, J.G. Lightner and H.J Siriwardane, Thin-layer element for interfaces and joints, Int J Num and Analyt Meth in Geomechanics 8 (1984), 19-43.

[14] N. Wagner and L. Gaul, Eigenpath analyses of friction induced vibrations depending on the friction coefficient, PAMM 3 (2003), $130-131$. 
[15] I. Bar-on and M. Paprzycki, High performance solution of the complex symmetric eigenproblem, Numerical Algorithms 18 (1998), 195-208.

[16] F. Cortes and M.J. Elejabarrieta, An approximate numerical method for the complex eigenproblem in systems characterised by a structural damping matrix, Journal of Sound and Vibration 296 (2006), 166-182.

[17] B.N. Parlett, The Symmetric Eigenvalue Problem, volume 20 of Classics In Applied Mathematics. SIAM, 1998.

[18] G. Peters and J.H. Wilkinson, Inverse iteration, ill-conditioned equations and newton's metho, SIAM Review 21(3) (1979), 339-360.

[19] J.H. Wilkinson, Some recent advances in numerical linear algebra, in: The State of the Art in Numerical Analysis, D. Jacobs, ed., Academic Press, London New York San Francisco, 1977, pp. 3-53.

[20] Françcoise Chatelin and Mario Ahués. Eigenvalues of Matrices, John Wiley, Chichester, 1993.

[21] A. Amiraslani and P. Lancaster, Rayleigh quotient algorithms for nonsymmetric matrix pencils, Numerical Algorithms 51 (2009), 5-22.

[22] Z. Bai, J. Demmel, J. Dongarra, A. Ruhe and H. van der Vorst, editors, Templates for the Solution of Algebraic Eigenvalue Problems: A Practical Guide. SIAM, Philadelphia, 2000.

[23] G.H. Golub and C.F. Van Loan, Matrix Computations, Johns Hopkins University Press, 1986.

[24] B.N. Parlett, The rayleigh quotient iteration and some generalizations for nonnormal matrices, Mathematics of Computation 28(127) (1974), 679-693.

[25] A. Ruhe, Computation of eigenvalues and eigenvectors, in: Sparse Matrix Techniques, V.A. Barker, ed., Springer-Verlag, 1977, pp. $130-184$. 

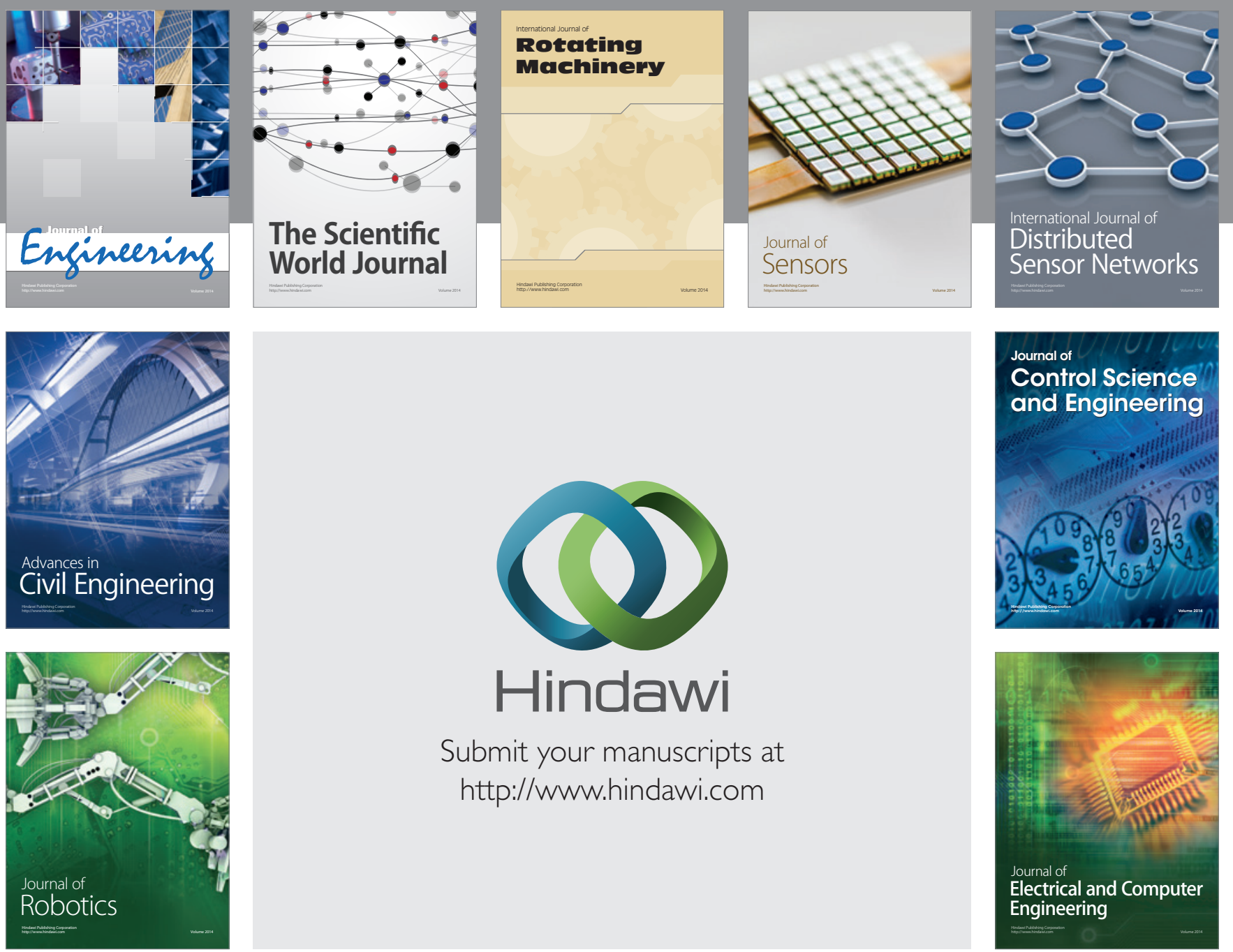

Submit your manuscripts at

http://www.hindawi.com
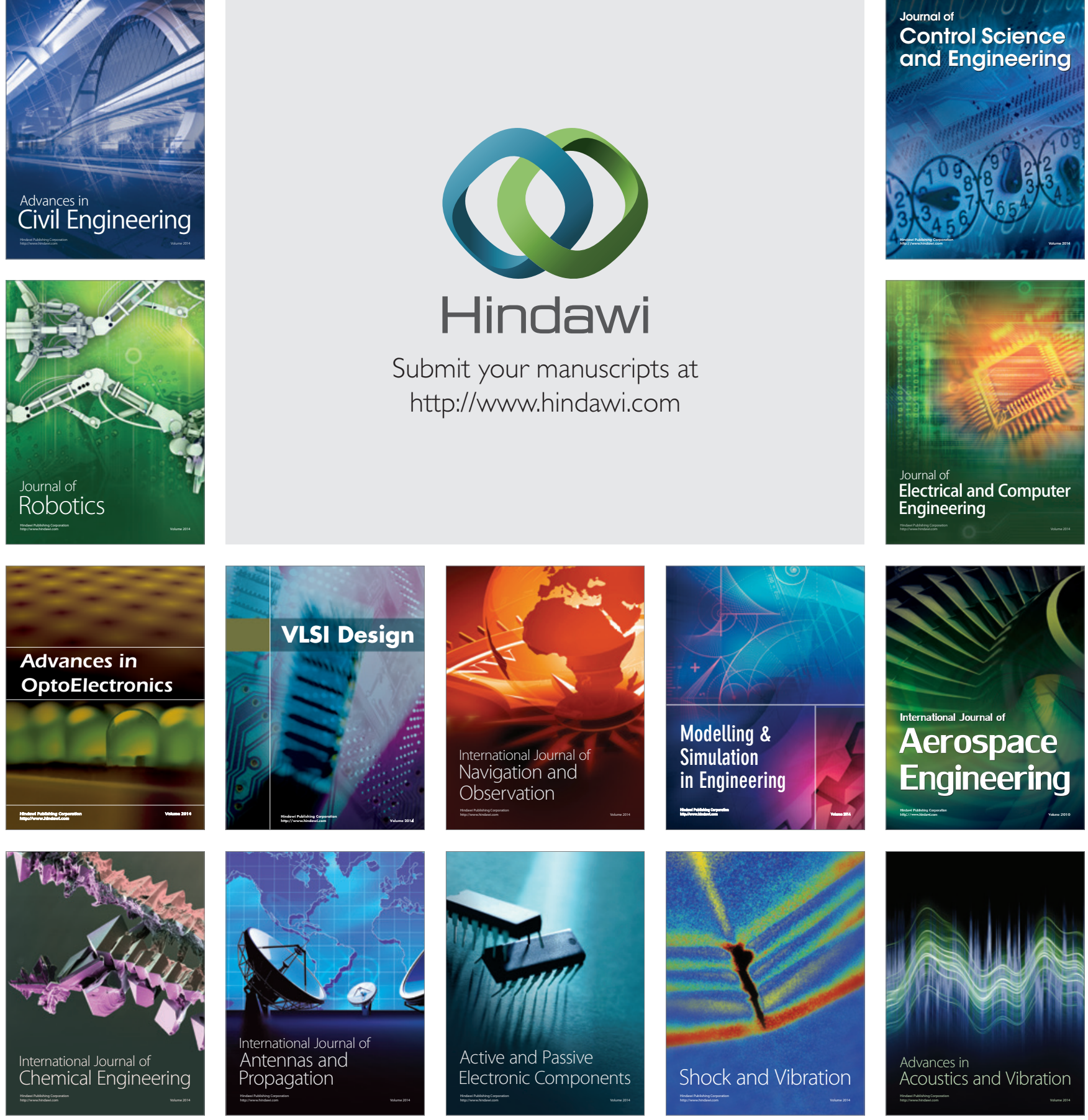